\title{
Evaluación de un inhibidor base nitritos en agua desmineralizada a $55^{\circ} \mathrm{C}$ a bajas concentraciones \\ Evaluation of a nitrite base inhibitor in demineralized water at $55^{\circ} \mathrm{C}$ at low concentrations
}

\author{
C. R. Arganis-Juárez ID a, *, J. C. Zenteno-Suárez iD a \\ ${ }^{a}$ Instituto Nacional de Investigaciones Nucleares, 52750 Ocoyoacac Estado de México, México
}

\begin{abstract}
Resumen
El nitrito de sodio se ha utilizado durante muchos años para inhibir la corrosión de los metales por el agua. Al funcionar como un inhibidor anódico. El nitrito de sodio oxida los iones ferrosos a iones férricos en las superficies de hierro y acero para producir una película adherente muy delgada de óxido férrico en las áreas anódicas. En el presente estudio se evalúa el comportamiento electroquímico de un inhibidor base nitrito, en agua desmineralizada, en un acero estructural con $0.21 \% \mathrm{C}$, en el intervalo de 0 a $800 \mathrm{ppm}$ de nitritos $\left(0.44,0.88,1.76,3.52\right.$ y $5.28 \mathrm{ml} / 1$ de Inhibidor) a $55^{\circ} \mathrm{C}$, mediante resistencia a la polarización (Rp), Ruido Electroquímico (EN) y curvas de polarización. Los resultados muestran que la presencia del inhibidor reduce la velocidad de corrosión, alcanzando un máximo a $0.88 \mathrm{ml} / 1$ pero vuelve a aumentar ligeramente al incrementar la concentración en el medio del inhibido.
\end{abstract}

\section{Palabras Clave:}

Inhibidores base nitrito, Corrosión en acero al carbono en agua desmineralizada, Técnicas electroquímicas.

\begin{abstract}
Sodium nitrite has been used for many years to inhibit water corrosion of metals. As an anodic inhibitor, sodium nitrite oxidizes ferrous ions to ferric ions on iron and steel surfaces to produce a thin cling film of ferric oxide on anodic areas. In the present study, the electrochemical behavior of a nitrite-based inhibitor is evaluated, in demineralized water, in structural steel with $0.21 \% \mathrm{C}$, in the range of 0 to $800 \mathrm{ppm}$ of nitrites $\left(0.44,0.88,1.76,3.52\right.$, and $5.28 \mathrm{~mL} / \mathrm{L}$ of inhibitor) at $55{ }^{\circ} \mathrm{C}$, through resistance to polarization (Rp), Electrochemical Noise (EN) and polarization curves techniques. The results show that the presence of the inhibitor reduces the corrosion rate, reaching a maximum of $0.88 \mathrm{~mL} / \mathrm{L}$ but increases again slightly with the increases in inhibitor concentration.
\end{abstract}

Keywords:

Nitrite Inhibidors, Corrosion of carbon steel in demineralized water, Electrochemical Techniques.

\section{Introducción}

Los generadores diésel de emergencia (EDG) se utilizan como fuente de respaldo de energía de emergencia en plantas de energía nuclear, alimentando sistemas de enfriamiento del núcleo y otros equipos necesarios para mantener el apagado seguro de los reactores de energía nuclear. (Suez 2021).

Esto requiere circuitos cerrados de agua de enfriamiento que permitan el arranque sin el uso de ventiladores de enfriamiento de aire, en el caso de una pérdida de energía externa. Viswanathan, 2010 ha mencionado que los EDG han presentado corrosión y fugas en las tuberías de agua de refrigeración. Los sistemas no tratados pueden sufrir graves daños por corrosión por picaduras de oxígeno, acción galvánica y ataque por grietas. Los sistemas de enfriamiento cerrados que se apagan periódicamente están sujetos a temperaturas del agua que pueden variar desde la temperatura ambiente hasta $180^{\circ} \mathrm{F}\left(82^{\circ} \mathrm{C}\right)$ o más. Durante el apagado, el oxígeno puede ingresar al agua hasta que se alcanza su límite de saturación. Cuando el sistema vuelve a funcionar a alta

*Autor para la correspondencia: carlos.arganis@inin.gob.mx

Correo electrónico: carlos.argani@inin.gob.mx (C.R. Arganis-Juàrez), juliocesar.zenteno@inin.gob.mx (J. C. .Zenteno-Suárez) 
temperatura, la solubilidad del oxígeno cae y el oxígeno liberado ataca las superficies metálicas (Viswanathan 2010)

Por otra parte, el nitrito de sodio se ha utilizado durante muchos años para inhibir la corrosión de metales por el agua (Beecher et al., 1959), y es un inhibidor de agua de refrigeración cerrado no cromado ampliamente aceptado. Las concentraciones de nitrito en el rango de 600-1200 ppm como $\mathrm{NO}_{2}^{-}$inhibirán adecuadamente la corrosión del hierro y el acero cuando el $\mathrm{pH}$ se mantenga por encima de 7.0. Los sistemas que contienen pares de acero y cobre requieren niveles de tratamiento en el rango de 5000 a 7000 ppm (Suez 2021)

Al funcionar como un inhibidor anódico, el nitrito de sodio oxida los iones ferrosos a iones férricos en las superficies de hierro y acero para producir una película adherente muy delgada de óxido férrico en las áreas anódicas. (Beecher et al., 1959)

El nitrito de sodio, es eficaz en soluciones acuosas con $\mathrm{NaCl}$ (Farooqi y Saini, 2000, Matsuda y Uhlig, 1964 y Mercer et al. 1968), principalmente inhibe la reacción anódica y forma una película protectora muy fina de óxido de $\mathrm{Fe}_{2} \mathrm{O}_{3}+\gamma-\mathrm{Fe}_{2} \mathrm{O}_{3}$ en la superficie del metal.

Hayyan et al, 2012, probaron que para soluciones de $\mathrm{NaCl}$ inhibidas y desinhibidas con concentraciones de cloruros de 250 ppm, en presencia de $\mathrm{NaNO}_{2}$ con diferentes concentraciones, la efectividad de la protección dependen de la relación molar $\left[\mathrm{NaNO}_{2} / \mathrm{NaCl}\right]$ y la frecuencia angular del electrodo rotatorio. Su análisis reveló que el nuevo método para predecir el valor de protección óptimo es más confiable que la técnica convencional basada en la densidad de corriente de corrosión.

En el presente trabajo se evaluó el efecto de la concentración de nitritos en un inhibidor comercial sobre acero estructural con $0.21 \% \mathrm{C}$, en bajas concentraciones de nitritos en agua desmineralizada, a $55^{\circ} \mathrm{C}$, con el fin de obtener datos para una posible protección. de un circuito cerrado de agua de refrigeración tuberías para un EDG

\section{Desarrollo Experimental}

\subsection{Materiales.}

Se utilizó una placa de acero al carbono cuya composición se muestra en la Tabla I, que corresponde a un acero al carbono con $0.21 \%$ en peso de Carbono (AISI 1020). La placa se cortó en cubos de $1 \mathrm{~cm}^{2}$ por cara. Los cubos se prepararon hasta lija 600, se limpiaron con etanol, se secaron y se embebieron en baquelita para realizar pruebas de corrosión por técnicas electroquímicas. La composición química se evaluó por un Espectrómetro Híbrido Portátil marca BELEC ${ }^{\mathrm{TM}}$.

Tabla 1. Composición Química del acero al carbono (\% peso)

\begin{tabular}{ccccccc}
\hline & $\mathrm{C}$ & $\mathrm{Si}$ & $\mathrm{Mn}$ & $\mathrm{Al}$ & $\mathrm{Cu}$ & $\mathrm{Cr}$ \\
\hline Steel & 0.21 & 0.07 & 0.45 & 0.062 & 0.06 & 0.04 \\
\hline
\end{tabular}

El inhibidor utilizado, fue un producto comercial a base de nitrito TRASAR TRAC102TM y la dosis utilizada fue la proporcionada por el proveedor. Se prepararon soluciones de un litro con $0.44,0.88,1.76,3.52$ y $5.28 \mathrm{ml} / 1$ de Inhibidor. En un trabajo previo (Arganis et. al 2019), se realizó la curva de calibración entre la dosificación y la concentración de nitrito de sodio en ppm mediante análisis por absorción atómica, encontrándose una relación lineal con coeficiente de correlación $\mathrm{R}=0.9994$ para la ecuación

$$
\text { Con }=70.456 \text { Dos }+7.586
$$

Donde Con es la concentración en ppm de Nitrito de Sodio y Dos es la dosificación en ml/l.

\subsection{Pruebas electroquímicas.}

Las muestras (Electrodo de trabajo (Etrab)) se ensayaron mediante la técnica de resistencia a la polarización y curvas de polarización por duplicado en agua desmineralizada y en soluciones con nitritos del inhibidor y las pruebas se realizaron a $55^{\circ} \mathrm{C}$. Se utilizó un barrido de -20 a $20 \mathrm{mV}$ vs. el potencial de corrosión electroquímico $\mathrm{E}_{\text {corr }}$ o potencial de reposo con una velocidad de barrido de $1 \mathrm{mV} /$ minuto para las pruebas de polarización lineal con un equipo Gill AC de ACM Instrument. Se utilizó un barrido de -250 a $250 \mathrm{mV}$, utilizando una velocidad de barrido de $1 \mathrm{mV} /$ minuto, para las curvas de polarización. En una celda de vidrio de cinco bocas, un electrodo de grafito como electrodo auxiliar (Eaux) y un electrodo de referencia (Eref) de plata / cloruro de plata $3.8 \mathrm{M}(0,199 \mathrm{mV}$ frente al electrodo de hidrógeno estándar (SHE)) fueron utilizados.

Para las pruebas de ruido electroquímico se introdujo un electrodo de Pt de $0.5 \mathrm{~mm}$ de diámetro y $2 \mathrm{~mm}$ de largo, como segundo electrodo de trabajo para el monitoreo de la densidad de corriente entre ambos electrodos y en el caso del potencial, este se midió entre el electrodo de trabajo y el electrodo de referencia de Plata/Cloruro de Plata. La medición de ambos parámetros se realizó con una frecuencia de $1 \mathrm{~Hz}$. La figura 1 muestra una fotografía del equipo utilizado para estas pruebas Las condiciones de laboratorio son $3.041 \mathrm{~m}$. de altitud sobre el nivel del mar y una presión de $534 \mathrm{~mm}$ de Mercurio y para mantener la temperatura se utilizó un baño con control de temperatura y una columna de enfriamiento para compensar la evaporación.

\section{Resultados y discusión.}

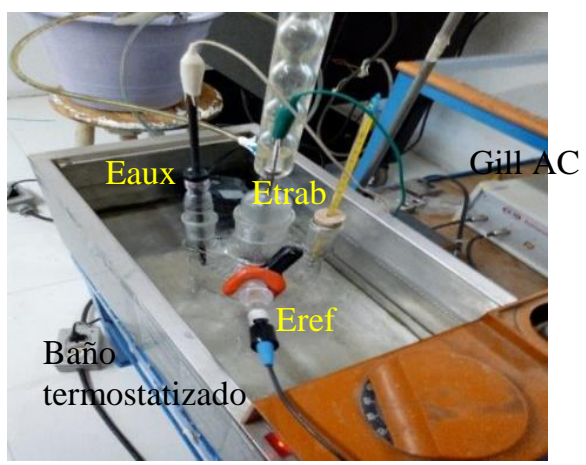

Figura 1 Equipo utilizado para realizar las pruebas electroquímicas en acero en agua desmineralizada a $55^{\circ} \mathrm{C}$ con las diferentes dosificaciones. 
La Figura 2 muestra las gráficas de polarización lineal realizadas al acero en agua desmineralizada a $55^{\circ} \mathrm{C}$, con diferentes dosificaciones de inhibidores.

La tabla 2 muestra los resultados de resistencia a la polarización RP, potencial electroquímico de corrosión $\mathrm{E}_{\text {corr }}$ densidad de corriente de corrosión $\mathrm{i}_{\text {corr }} \mathrm{y}$ velocidad de corrosión (Vcorr) obtenidas en las diferentes dosificaciones de inhibidor.

La resistencia a la corrosión RP fue obtenida por la pendiente en las curvas de polarización lineal $(\Delta \mathrm{E} / \Delta \mathrm{i})$ en la porción lineal cera del Ecorr y la Vcorr fue obtenida mediante la ecuación (Stern y Geary 1957)

$$
\operatorname{Vorr}=\frac{k \cdot B}{R P}
$$

Dónde B es la constante de Stern y Geary 1957, relacionada con las pendientes de Tafel anódicas y catódicas y se consideró las pendientes de Tafel de $120 \mathrm{mV} /$ década, $\mathrm{k}$ es la constante que relaciona la densidad de corriente de corrosión icorr y la Vcorr aplicando la ley de Fraday. K=11.6 ( $\mu \mathrm{m} / \mathrm{año}) /(\mu \mathrm{A} / \mathrm{cm} 2)$

Tabla 2. Resultados obtenidos mediante la técnica de Polarización Lineal y dosificación y concentración de nitritos obtenidas.

\begin{tabular}{|l|l|l|l|l|l|}
\hline $\begin{array}{l}\text { Dosifi } \\
\text { cación } \\
\mathrm{ml} / 1\end{array}$ & Conc & $\begin{array}{l}\mathrm{E}_{\text {corr }} \\
(\mathrm{mV})\end{array}$ & $\begin{array}{l}\mathrm{RP} \\
\text { Ohms.c } \\
\mathrm{m}^{2}\end{array}$ & $\mathrm{i}_{\text {corr }} \mathrm{mA} / \mathrm{cm}^{2}$ & $\begin{array}{l}\text { Vel. de } \\
\text { Corrosión. } \\
(\mathrm{mm} / \mathrm{año})\end{array}$ \\
\hline 5.28 & 300 & -273.16 & 412480 & $63.24 \times 10^{-00}$ & $0.7 \times 10^{-5}$ \\
\hline 3.52 & 200 & -307.35 & 242320 & $107.7 \times 10^{-00}$ & $1.27 \times 10^{-3}$ \\
\hline 1.76 & 100 & -346.77 & 1960000 & $13.31 \times 10^{-00}$ & $0.15 \times 10^{-3}$ \\
\hline 0.88 & 50 & -293.39 & 4517800 & $5.774 \times 10^{-00}$ & $66.92 \times 10^{-0}$ \\
\hline 0.44 & 25 & -256 & 2260600 & $11.54 \times 10^{-00}$ & $0.13 \times 10^{-5}$ \\
\hline 0 & 0 & -547.8 & 41730 & $624.9 \times 10^{-00}$ & 0.0691 \\
\hline
\end{tabular}

La Figura 3 muestra las curvas de polarización obtenidas para el acero con las diferentes concentraciones/dosificaciones del inhibidor. Es posible ver un efecto el potencial electroquímico de corrosión $\mathrm{E}_{\text {corr }}$ que se desplaza a valores más positivos, pero este empieza a disminuir con la adición de más nitritos aumentar. Las curvas se desplazan hacia la izquierda, proporcionando una densidad de corriente anódica más pequeña. A partir de 100 ppm de nitritos, las curvas anódicas están desplazadas a la derecha con respecto a las curvas de 25 y 50 ppm, pero están más a la izquierda que la curva sin nitratos. Tal vez este efecto se deba a que el $\mathrm{NO}_{2}^{-}$es un ion reductor y en exceso puede desestabilizar la capa de $\mathrm{Fe}_{2} \mathrm{O}_{3}$ a $\mathrm{Fe}_{3} \mathrm{O}_{4}$ (Magnetita) que no tiene las mismas propiedades pasivantes. Se requieren por supuesto aplicar otro tipo de técnicas de análisis para poder comprobar esta posibilidad.

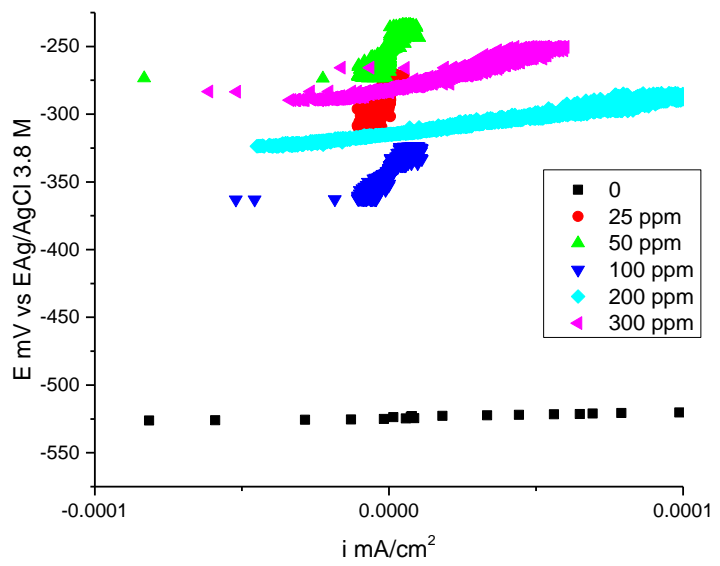

Figura 2 Pruebas de Polarización Lineal del acero en agua desmineralizada a $55^{\circ} \mathrm{C}$ a diferentes concentraciones de 0 a 300 ppm de nitritos.

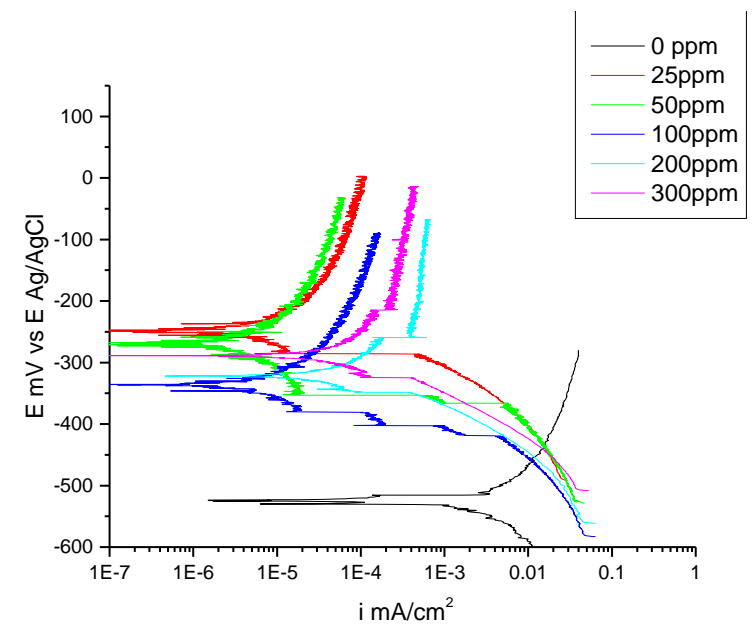

Figura 3 Curvas de Polarización del acero en agua desmineralizada a $55^{\circ} \mathrm{C}$ con diferentes concentraciones de 0 a $300 \mathrm{ppm}$ de nitritos.

La figura 4 muestra las series de tiempo de ruido electroquímico en corriente, para las diferentes concentraciones

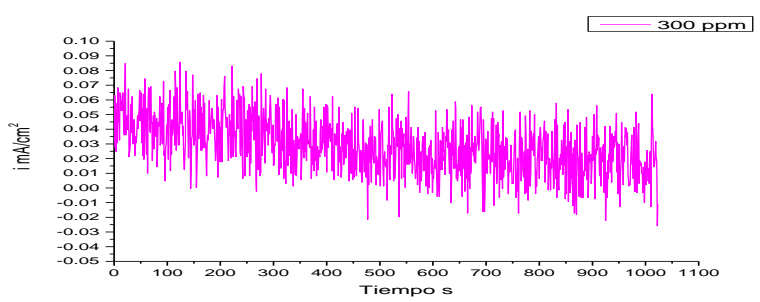



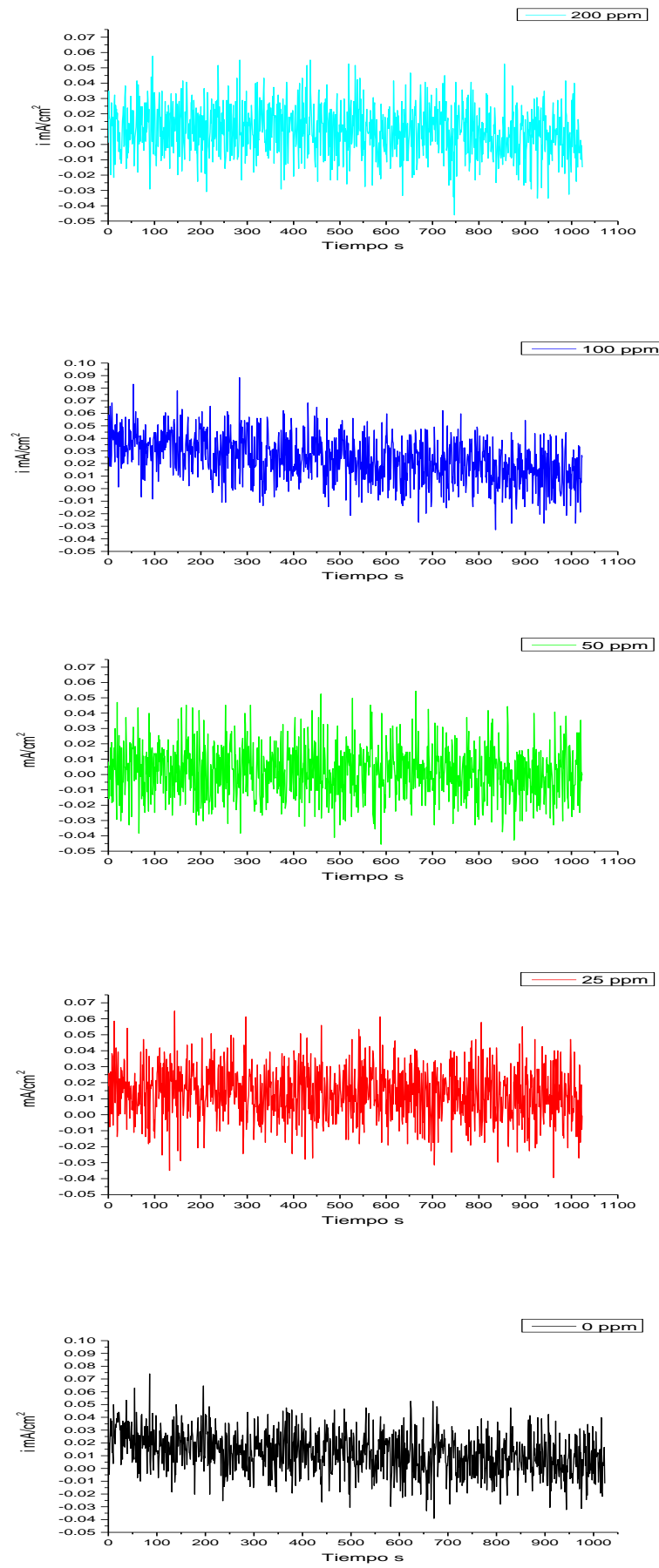

Figura 4 Series de tiempo de ruido electroquímico en corriente del acero en agua desmineralizada a $55^{\circ} \mathrm{C}$ con diferentes concentraciones de 0 a 300 ppm de nitritos.

Las series de tiempo en voltaje se presentan en las figuras 5. En este caso se observaron algunos desplazamientos del potencial de corrosión, por lo que fue necesario hacer una remoción de tendencia con un polinomio de cuarto grado. En la solución sin inhibidor, existen transitorios de cierta duración hacia la dirección positiva y luego el regreso a un valor base. Al agregar el inhibidor los transitorios cambian a transitorios rápidos en ambas direcciones alrededor de un valor base, hasta la concentración de inhibidor de 100 ppm. A partir de este valor, vuelven los transitorios similares a la solución sin inhibidor
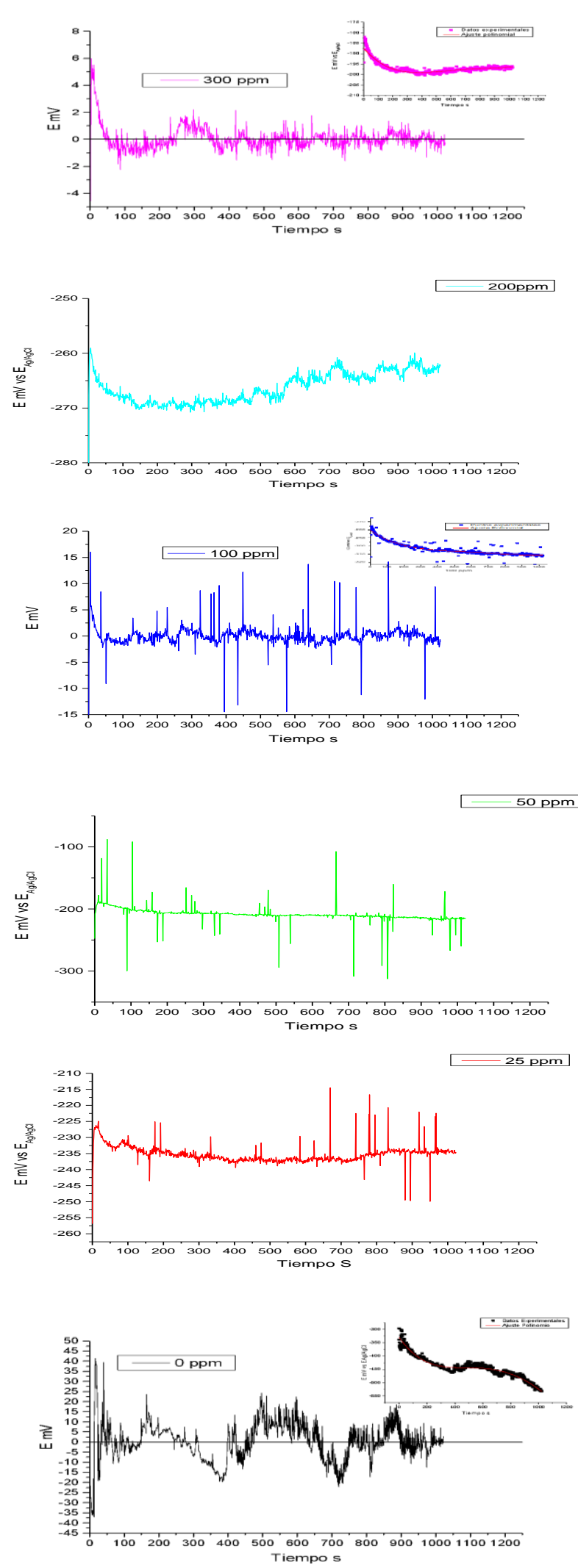

Figura 5 Series de tiempo de ruido electroquímico en voltaje del acero en agua desmineralizada a $55^{\circ} \mathrm{C}$ con diferentes concentraciones de 0 a 300 ppm de nitritos.

Se obtuvieron las desviaciones estándar en corriente, $\sigma i$ y voltaje $\sigma E$ para obtener la resistencia a la polarización de ruido 
electroquímico $R P_{E N}$ mediante la ecuación (Eden y Rothwell, 1992).

$$
R P_{E N}=\frac{\sigma E}{\sigma i}
$$

Y se utilizó el índice de Localización $L I$, mediante la siguiente ecuación, dónde la irms es la raíz medía cuadrática de la densidad de corriente (Eden y Rothwell 1992).

$$
L I=\frac{\sigma i}{i r m s}
$$

La tabla 3 muestra los resultados obtenidos en análisis de las series de tiempo de densidad de corriente y voltaje. Los procesos de corrosión uniforme pueden ser identificados por diversas técnicas, en primera instancia el registro de datos en el tiempo de este tipo de procesos exhibe pocos signos de eventos individuales no-correlacionados, y por lo tanto hay pocos si no es que ningún transitorio rápido. (Eden y Rothwell, 1992). En la figura 4 es posible observar que las series de tiempo en densidad de corriente no presentan transitorios rápidos de corrosión localizada, si no oscilaciones alrededor de un punto central, por

\begin{tabular}{|c|c|c|c|c|}
\hline $\begin{array}{l}\text { Conc } \\
\text { ppm. }\end{array}$ & $\begin{array}{l}\text { Desv. } \\
\text { Estándar } \\
(\mathrm{mV})\end{array}$ & $\begin{array}{l}\text { Desv } \\
\text { Estándar } \\
\text { i } \mathrm{mA} / \mathrm{cm}^{2}\end{array}$ & \begin{tabular}{|l|}
$\mathrm{RP}_{\mathrm{EN}}$ \\
$\mathrm{Ohms.cm}^{2}$
\end{tabular} & LI \\
\hline 300 & $2.063 *$ & 0.0191 & 108.01 & 0.559 \\
\hline 200 & 2.890 & 0.0160 & 179.53 & 0.872 \\
\hline 100 & $2.186^{*}$ & 0.0183 & 119.45 & 0.614 \\
\hline 50 & 12.569 & 0.0167 & 751.71 & 0.995 \\
\hline 25 & 2.754 & 0.0162 & 169.33 & 0.777 \\
\hline 0 & $9.567 *$ & 0.0163 & 584.01 & 0.807 \\
\hline
\end{tabular}
lo que se pueden atribuir a corrosión uniforme.

Tabla 3. Resultados obtenidos de desviación estándar en potencial y densidad de corriente, $\mathrm{RP}_{\mathrm{EN}}$ e LI mediante la técnica de ruido electroquímico.

*Remoción de tendencia con polinomio de cuarto grado.

La $\mathrm{RP}_{\mathrm{EN}}$ es de ordenes de magnitud menor que la RP obtenida por la técnica de polarización lineal. El ensayo sin inhibidor presenta una $\mathrm{RP}_{\mathrm{EN}}$, mayor, lo que podría traducirse que las pruebas utilizando ruido electroquímico, la presencia del inhibidor aumenta la velocidad de corrosión, pero con la dosificación de $0.88 \mathrm{ml} / \mathrm{l}$ que corresponde a la concentración de 25 ppm, dónde

la $\mathrm{RP}_{\mathrm{EN}}$ es mayor y por lo tanto su velocidad disminuye. En este valor, coincide en ambas técnicas cómo el valor de mayor protección a la corrosión.

Los índices de localización en ruido electroquímico en densidad de corriente muestran un comportamiento un tanto aleatorio. Lo que permanece aproximadamente constante es la desviación estándar en densidad de corriente en todos los casos.
Como puede observare en la tabla 3, los índices de localización son mayores a 0.1 y de acuerdo a Eden y Rothwell, 1992 , estarían asociados a un proceso con corrosión localizada, sin embargo, debido a que no se presentan transitorios aislados, asociados a rompimiento de película y repasivación de la misma, en las series de tiempo en densidad de corriente, parece que este es un caso de falso positivo como los mencionados por Mansfield y Sun, 1999 cuando la irms es muy cercana a cero y la desviación estándar en densidad de corriente es del mismo orden de magnitud que la irms.

La eficiencia de inhibición EI, se obtuvo utilizando la ecuación 5, en dónde RP es la resistencia a la polarización del acero al carbono y $\mathrm{RP}_{\text {in }}$ es la resistencia a la polarización del acero al carbono con el inhibidor

$E I=\frac{R P_{\text {in }}-R P}{R P_{\text {in }}} * 100 \ldots \ldots \ldots$

La tabla 4 muestra los resultados de eficiencia de inhibición y los valores de Curtosis obtenidas en las series de tiempo en densidad de corriente y en potencial. De acuerdo a Cottis y Turgoose, 1999, valores mayores a 5 están asociados a casos que exhiben cambios espontáneos en la distribución de la amplitud, y cambios repentinos en la velocidad de corrosión

Tabla 4: Resultados de eficiencia de inhibición mediante RP

\begin{tabular}{|l|l|l|l|l|}
\hline Dosificación & $\begin{array}{l}\text { Conc } \\
\text { ppm. }\end{array}$ & $\begin{array}{l}\text { EI } \\
\%\end{array}$ & $\begin{array}{l}\text { Curtosi } \\
\text { en i }\end{array}$ & $\begin{array}{l}\text { Curtosi } \\
\text { en E }\end{array}$ \\
\hline 5.28 & 300 & 89.88 & -0.252 & 3.11 \\
\hline 3.52 & 200 & 82.77 & -0.097 & 2.89 \\
\hline 1.76 & 100 & 97.87 & -0.169 & 7.59 \\
\hline 0.88 & 50 & 99.07 & -0.055 & 38.20 \\
\hline 0.44 & 25 & 98.15 & -0.056 & 14.06 \\
\hline 0 & & & & \\
\hline
\end{tabular}

El Inhibidor TRASAR TRAC102TM en la dosificación de $0.88 \mathrm{ml} / \mathrm{l}$, concentración de $50 \mathrm{ppm}$ de nitrito tiene la mayor eficiencia de inhibición de $99.07 \%$, disminuyendo si se agrega mayor concentración de este, en el intervalo estudiado. Esto concuerda también con la máxima $\mathrm{RP}_{\mathrm{EN}}$ obtenida con la técnica de ruido electroquímico.

Los bajos valores de curtosis en las series de tiempo de densidad de corriente, sugieren que se trata de un caso de 
corrosión uniforme. Las series de tiempo en potencial si tienen valores mayores a 5 y se podría asociar a cambios en la velocidad de corrosión, la cual concuerda con los transitorios observados en las mismas, pareciera que se asocia a inestabilidad de la película formada por el inhibidor la cual se estabiliza a mayores concentraciones de inhibidor.

\section{Conclusiones}

El Inhibidor TRASAR TRAC102 ${ }^{\mathrm{TM}}$ en la dosificación de $0.88 \mathrm{ml} / \mathrm{l}$, concentración de $50 \mathrm{ppm}$ de nitrito tiene la mayor eficiencia de inhibición de $99.07 \%$, disminuyendo si se agrega mayor concentración de este, en el intervalo estudiado.

Los valores de $\mathrm{RP}_{\mathrm{EN}}$ son de ordenes de magnitud menores a los obtenidos por polarización lineal, pero sus resultados concuerdan con la polarización lineal, al mostrar la máxima resistencia a la corrosión en la dosificación de $0.88 \mathrm{ml} / \mathrm{l}$ y concentración de 50 ppm de nitritos.

La naturaleza del tipo de corrosión no es posible determinarla por la técnica de ruido electroquímico ya que en las series de tiempo en densidad de corriente parece una corrosión uniforme, aun cuando los índices de localización tienen valores mayores a 0.1 , pero en las series de tiempo en potencial tiene ciertos transitorios y valores de curtosis mayores a cuatro.

\section{Agrdecimientos.} ININ

Este trabajo fue patrocinado por el proyecto SN-06 (2020) de

\section{Referencias.}

Arganis Juárez C. R., Zenteno Suárez J.C., and Cárdenas F. (2019). Determinación de la dosificación de un inhibidor base nitritos en agua desmineralizada a $55^{\circ} \mathrm{C}$ en acero al carbono mediante técnicas electroquímicas y pérdida de peso. 34 congreso nacional de la sociedad mexicana de electroquímica SMEQ, Querétraro, Queretaro México.

Beecher J., Dinkel C. and Corwin S. (1959) Corrosion Inhibition with Sodium Nitrite, Journal American Water Works Association, Vol. 51, No. 9 1175-1180.
Cottis R. , Turgoose S. (1999) Corrosion Testing Made Easy. Electrochemical Impedance and Noise. Syrett B.C. Editor. NACE.

Eden D. A. and Rothwell A.N (1992) Corrosion 92 the NACE Annual Conference and Corrosion Show. , Paper No. 292. Electrochemical Noise data: Analysis interpretation and presentation Houston Texas USA

Farooqi I.H., Saini P.A. , Quraishi M A. (2000) Recent Trends In Cooling Water lnhibitors, Corrosion 2000, Paper No. 00332, NACE, Houston Texas USA.

Hayyan M., Sameh S. A. , Hayyan A., AlNashef I. M. (2012) Utilizing of Sodium Nitrite as Inhibitor for Protection of Carbon Steel in Salt Solution, Int. J. Electrochem. Sci. 7, $6941-6950$

Joseph C.P. (1971). Third Eur. Symp. Corr. Inhibitor, University of Ferrara, P.791.

Mansfield F. And Sun Z. (1999) Technical Note: Localization Index Obtained from Electrochemical Noise Analysis. Corrosion Vol. 55, No. 10 pp. 915-918, DOI: /10.5006/1.3283926

Matsuda S. and. Uhlig H. H. (1964). Effect of pH, sulfates, and chlorides on behavior of sodium chromate and nitrite as passivators for steel J. of Electrochem. Soc., 111, 156-161.

Mercer A. D. Jenkins, I.D. and Rhoades-Brown J.E. (1968). , Comparative study of factors influencing the action of corrosion inhibitors for mild steel in neutral solution: III. Sodium nitrite Br. Corr. J., 3, 136-144.

DOI: $10.1179 / 000705968798326217$

Stern M. and Geary L. (1957) Electrochemical Polarization: I. A Theoretical Analysis of the Shape of Polarization Curves J. of the Electrochemical Society, 104, 56-63

Suez water technologies \& solutions. (2021) Handbook of Industrial Water Treatment. Suez water Technologies \& Solutions. https://web.archive.org/web/20210623212126/https://www.suezwatertechnol ogies.com/handbook/chapter-32-closed-recirculating-cooling-systems.

Viswanathan S.S.,(2010) Contemporary Developments in Corrosion Inhibitors - Review of Patents. Recent Patents on Corrosion Science 2, 6-12. DOI:10.2174/1877610801002010006 